\title{
Theo Rasehorn Von der Klassenjustiz zum Ende der Justiz
}

Institutionen und Berufe haben kein ewiges Leben. Auch ein "Urberuf « verliert bei einem bestimmten Gesellschafts- und Bewußrseinszustand seine Lebenskraft. Jedem Einsichtigen ist klar, daß z. B. der Urberuf des Kriegers und Soldaten heute keine echte Chancen mehr besitzt. Liegt auch der Beruf des Richters im Sterben? Meine Zweifel an seinen Zukunftschancen haben sich durch die Ausführungen von Rottleuthner verstärkt. ${ }^{1}$ Bis zur Gewißheit, soweit es um die Personalunion von Richter und Justizbeamte geht. Die Justiz ist gewiß nicht mehr zu retten; der Richter vielleicht noch, vermag er sich vom Justizapparat zu lösen.

\section{Die Rollenkonflikte des Richters}

Bevor ich mich mit der Zukunft befasse: zur Justiz, zum Richter der Gegenwart. Hier sind die Ausführungen von Rottleuthner zu ergänzen und zu vertiefen; teilweise ist ihnen aber auch zu widersprechen. Die Vielschichtigkeit im Handeln und Bewußtsein des heutigen Richters, in den Strömungen der Justiz, insbesondere bei den Reformern, kommt bei ihm nicht so recht zum Vorschein. Bei der Tätigkeit des Richters von heute besteht eine Gemengelage von archaischen Determinierungen - der skönigliche Richter - vorwiegend allerdings von ordnungs- und machtpolitischen Zügen, mit denen sich Rottleuthner fast ausschließlich befaßt, wie aber auch von sozialpsychologischen. Der Rollenkonlikt im Richter ist permanent, so permanent wie wohl bei keiner anderen Berufsgruppe von vergleichbarer Größe.

Damit soll nicht unter einem neuen Vorzeichen das hohe Lied der hehren Richtertugenden gesungen werden. Rottleuthner hat die Selbstbeweihräucherung der Richter zu recht gegeißelt (und auch zu recht mir meine volltönenden, aber inhaltsleeren Worte zum Richterkittel» vorgehalten, S. I 8 ff.). Eine gründliche berufssoziologische Untersuchung mag ergeben, daß die von Richtern so herausgestrichenen vagen Persönlichkeitswerte für viele Berufe in gleicher Weise gelten. Zwar kann fast jeder Richter von dem Vorsitz in einem Auschwitz - oder Conterganprozeß getroffen werden; aber letztlich ist diese Möglichkeit so gering wie ein Blitzschlagtod. Im übrigen dürfte sicher sein, daß der Arzt, der oft in kurzen Augenblicken über das Leben des Patienten zu entscheiden hat, mehr Verantwortung tragen muß, und daß der Lehrer, der jeden Werktag und nicht nur zweimal in der Woche "Sitzung « hat, nervlich belasteter ist als der Richter.

Die besondere Rollenerwartung indes, die die Rechtssuchenden an den Richter stellen - ohne deren Erfüllung es zu keiner Kommunikation kommen kann - 
dürfte doch als typisches Berufserschwernis zu werten sein, das um so größer ist, je mehr sich Richter und Gesellschaft in einer Zeir des Ubergangs wie der heutigen befinden. Sicherlich ist es auch Aufgabe des Richters, an dern Erziehungsprozeß zur Mündigkeit mitzuwirken. Gerade dies vermag er bei einer großen Schicht von Rechtssuchenden nur, wenn er zunächst, ihren Erwartungen entsprechend, in den Mantel des «königlichen Richters« schlüpft. Noch größer ist die Zahl der Rechtssuchenden, die durch den Richter - sagen wir es ganz schlicht, aber um so mißverständlicher - betreut werden wollen. Diese Rollenerwartung bedeutet also nicht nur ein psychologisches, sondern auch ein gesellschaftliches Problem.

Sicher aber ist - und dies führt Rottleuthner an verschiedenen Beispielen aus (S. 7 ff.) -, daß der Durchschnittsrichter diesen Erwartungen nur zu einem geringen Teil gerecht zu werden vermag, da $B$ er aufgrund Herkommen, Erziehung, Ausbildung und ständiger Beeinflussung durch Justizapparat, höchstrichterliche Rechtsprechung und Iehre seine Aufgabe in der Aufrechterhaltung der Herrschaft ("Rechtsordnung ") sieht und dabei statt des kommunikativen Mitempfindens dem Rechtssuchenden gegenüber nur die Leutseligkeit des Höhergestellten zum Ausdruck bringt.

Noch komplizierter wird der Rollenkonflikt für den Reformer unter den Richtern. Er kommt, insbesondere als Mitglied eines Kollegiums, kaum daran vorbei, sich der »herrschenden Meinung " anzuschließen und Kompromisse mit Verhaltensformen einzugehen, die er ablehnt, will er nicht jeglichen Einfluß in seinem Beruf verlieren, will er vor allem nicht eine zu Lasten der Rechtssuchenden gehende Verhärtung hervorrufen.

Der progressive richterliche Praktiker ist insbesondere Rechts- und Justizpolitiker. Hierbei hat er wieder zwei Rollen zu übernehmen; einmal, das Bewußtsein seiner Kollegen zu beeinflussen, mitzuhelfen, den cultural lag der Justiz zu überwinden; zum anderen, gegenüber Gesetzgebung und Exekutive, gegenüber der Offentlichkeit für praktische Reformen einzutreten, bisweilen für Iächerlichkeiten (wie die Anrede ohne Dienstgrad), oft sogar für schlechte Lösungen, weil sie von den angebotenen Möglichkeiten die am wenigsten schlechten sind - auf sich zu nehmen die Bitterkeit des schlechten Kompromisses, des schlechten Gewissens, wie es die Aufgabe eines verantwortungsvollen Politikers in einer gespaltenen Zeit und in einer gespaltenen Gesellschaft ist. ${ }^{2}$ Von dieser Sicht aus werden auch manche Marksprüche über den Richter verständlich, hinter denen zwar, wie Rottleuthner hervorhebt, wenig steckr, die aber im öffentlich-politischen Raum allein durch ihr Vorhandensein Signale und Zeichen setzen können. ${ }^{3}$

Eine Rolle liegt uns allerdings fern: die des Justiztheoretikers. Wird darum unser Band Im Namen des Volkes? \& auf seine Justiztheorie abgeklopft - und hierbei neben vielem Tadelswerrem zu unserer Uberraschung auch Löbliches gefunden ${ }^{4}-$ so können wir nur darauf verweisen, daß wir keine Theorie haben entwidkeln wollen - vielleicht auch picht können; aber gewiß nicht wollen. Dennoch fühle ich mich geschmeichelt, wenn mich Rottleuthner in Schutz nimme, ich sei nicht völlig theorielos (S. 2I) $)^{5}$, und mir also einen Stehplatz im Theoretikerhimmel

\footnotetext{
2 Richtig ist aber, daß heute noch immer derjenige als skluger . Policiker gilt, der von vornherein auf den Kompromiß und das Ausklammem zielt.

3 Fairerweise müssen auch die besonderen Erschwernisse für den Richterreformer Wassermann herausgestell werden, der noch zusätzliche Rollen im Justiz- und Offentlichkeitsestablishment übernehmen muß.

4 Lenk Kritische Jusriz 1969, 107.

5 Anders R. Edkertz, Fünf Thesen zur Reform des juristischen Studiums Kritische Justiz 1968, I64, Anm. I4: \die (leider völlig theorielose) Kritik des... Xaver Berra «; Lenk, a.a.O., \$Feh-
} 
dem ein für den Richter fremdes Rechtsgebiet, bei dem nicht nur strafrechtliche, sondern auch verfassungsrechtliche und soziologische Tatbestände zu berücksichtigen sind; sie haben es mit einem in der Tateinstellung nicht geläufigen Tätertyp zu tun und mit Angeklagten, die die prozessualen Spielregeln nicht einhalten wollen, die es nicht auf ein geneigtes Verhalten des Richters, auf geringe Strafen, abstellen, sondern ihn gerade provozieren, umfunktionieren wollen. Dies muß den Durchschnittsrichter vor nahezu unlösbare Aufgaben stellen, weshalb hier die psychologische Problematik größer als die gesellschaftliche zu sein scheint.

Aber gerade darum entsteht bei Rottleuthner ein Zerrbild, wenn "kleine« Richter wie Schwalbe, Möller, Röhr, Pietsch als Exponenten der Richterherrschaft erscheinen. Dies mag aus der Sicht der angeklagten Demonstranten zutreffen; aber nur in der gleichen oberflächlichen Weise wie dem streikenden Arbeiter im Wilhelminischen Staat im Schutzmann die Repression ausübende Staatsgewalt erschien. Die "Schreibrischtäter" kommen einmal mehr glimpflich davon. Es wird nicht berücksichtigt, daß Amtsrichter und Schutzmann unten auf der Leiter stehen, sie wirken zwar repressiv, sind aber zugleich ein Opfer der Repression.

Damit steht nicht im Widerspruch, daß auch die Richter der Eingangsstufe ihrer Herkuntt und Erziehung nach vorwiegend zur Oberschicht (besser: obere Mittelschicht) gehören. Wie sehr es reines Wunschdenken ist, eine andere soziologische Zusammensetzung der jüngeren Richterschaft zu prognostizieren, wie neuerdings v. Münchhausen ${ }^{13}$, zeigen die Arbeiten von Richter und vor allem die zur Zeit erscheinende große Juristenanalyse von Kaupen ${ }^{14}$. Die Erfahrungen und Beobachtungen von Liebknecht, die Rottleuthner verdienstvollerweise der Vergangenheit entrissen hat (S. 7), haben darum noch aktuellen Bezug.

Herkommen und Erziehung des Richrers sind natürlich nur eine Komponente für seine Einstellung zur Gesellschaft, weshalb auch Rottleuthner zu recht seine Einbindung in den Justizapparat sowie die »rechtswissenschaftliche Sozialisation hervorhebt (S. I6 ff.). Während er den Einfluß des Justizapparats zutreffend schildert - hier wird eine in Bälde erscheinende Auswertung einer Fragebogenerhebung des Kölner soziologischen Instituts neue Erkennenisse bringen ${ }^{15}$ - unterschätzt er m. E. den Einfluß der Rechtsdogmatik auf die Rechtsprechung und das Verhalten des Richters erheblich.

Zwar legt er richtig dar, daß das Spezifische gerade des strafrechtlichen Tatbestands - die spraktikable Reduktion auf Wissen und Wollen eines personalen Täters $\propto$, wonach aus einem Film von tausend Bildern ("Lebenssachverhalt sagen wir Praktiker verschwommen) eines herausgegriffen und als allein maßgeblich erklärt wird - in den Fallösungstechniken unserer juristischen Dogmatiker seine Wurzel hat (wobei dennoch Rottleuthner dem Richter hierfür die Verantwortung auferlegt - S. 22). Weiter erweisen sich besonders aufschlußreich und zutreffend seine Ergebnisse im methodologischen Ansatz, unsere Methodenlehre verkenne, wie sehr für eine richrerliche Entscheidung die Handlungssituation mit ihren kommunikativen Argumenten maßgebend sei, und gehe deshalb zu Unrecht von dem einsamen Richter aus (S. 3). Dies wäre noch schärfer zu pointieren: nicht vom Richter geht sie aus, sondern von dem Abstraktum Rechtsprechung, als sei der Umstand, daß dahinter ein Mensch stehe, anstößig, wie für gewisse scholastische Theorien die Zeugung des Lebens durch den Geschlechtsverkehr.

13 Der Richter in der Gesellschaft, Recht und Politik 1969, 26.

14 Hüter von Recht und Ordnung, Neuwied 1969.

15 Kaupen-Rasehorn, Die Justiz zwischen Obrigkeitsstaat und Demokratie, wird Mitte r970 bei Luchterhand erscheinen. 
Aber wenn die Richter, die richterlichen Praktiker, gerade dieser - auch von Rottleuthner als falsch und unheilvoll bezeichneten Methodologie so uneingeschränkt, wenn auch im Zivilprozeß oft nur zum Schein, folgen, so wird dies doch weniger mit Herkommen und Erziehung des Richters oder mit seiner Abhängigkeit vom Justizapparat als vielmehr mir einem durchschlagenden Einfluß der Rechtsdogmatik zusammenhängen. Man mag mich hier, der ich diesen Punkt stets besonders herausstelle ${ }^{18}$, einer gewissen Einseitigkeit zeihen; aber es gibt objektive Gründe, anzunehmen, daß eine Untersuchung über Theorie und Praxis der akademischen Berufszweige in Deutschland zu dem Ergebnis führen wird, daß dieses Verhältnis auf dem Gebiet des Rechtswesens am gestörtesten ist. Bei der Medizin, bei den technischen Berufen ergibr sich eine Klammer daraus, daß der Hochschullehrer zugleich als Arzt (Privar-)Patienten behandelt, als Architekt Bauten errichtet usw. Für die Philologen gilt die völlige Trennung - der Professor hat mit dem Unterricht, den die von ihm ausgebildeten Studienräte geben, kaum noch etwas zu schaffen. Der Rechtslehrer hingegen beeinflußt gerade in seiner Eigenschaft als Theoretiker die Praxis unmittelbar, jedenfalls deren Spitze, die höchstrichterliche Rechtsprechung. Vorwiegend mit der Meinung von Autoren und nicht von Gerichten setzt sich der BGH auseinander ${ }^{17}$, ein Verhalten, das im englischen Recheswesen undenkbar ist ${ }^{18}$. Obskur ist es jedenfalls, wenn noch heute unsere Dogmatiker von der »juristischen Ästhetik « sprechen - Bemühen um Recht und Gerechtigkeit für Mensch und Gesellschaft als Glasperlenspiel'19! Aber Ästhetizismus wäre noch harmlos - tatsächlich geht es um Macht. Unter dem Vorwand, Recht und Gerechtigkeit seien das höchste Gut unserer Demokratie, wird ein technisch juristischer Perfektionismus propagiert, dessen gesellschaftlich-ökonomische Effizienz höchst zweifelhaft, dessen undemokratische Auswirkung hingegen evident ist. Notwendige Regelungen gesellschaftlicher Vorgänge werden, zu juristischen Spezialproblemen verfremdet, mit einem Knäuel perfektionistischer Normen bis zur Unkenntlichkeit umgarnt ${ }^{20}$, womit die Gesetzgebung unter die Herrschaft einer Handvoll Spezialisten gerät, die mit ihrer „Sachkennenis « den Bundestag dupieren. In der Herrschaft der dogmatischen Jurisprudenz zeigte sich schon vorzeirig das Modell von Herbert Marcuse für eine Technokratie: der Einsatz von Machtmitteln auf odemokratische « Weise, über Werbung und Reklame, über öffentliche Beeinflussung durch das Ausbildungswesen und die Rechtspresse - die ja zu $90 \%$ in den Händen der Rechtsdogmatik liegt - und über repressive Toleranz gegenüber »abwegigen« Theorien.

Indessen ist das Nachlassen der Liberalität evident. Die gleiche Haltung, die Rechtslehrer wie Larenz, Forsthoff, Maunz, die der Hitlerei Elogen gewidmet haben ${ }^{21}$, zu Koryphäen ihrer Fachgebiete aufrücken läßr, vereitelt umfassende

16 Berra, a. a. O., S. 70; Rasehorn, Wege einer Soziologie des Richters, Kölner Zeirschritt für Soziologie und Sozialpsychologie 1968, 1ro; Was formt den Richter? in Böhme, Weltanschauliche Hintergründe in der Rechtsprechung, Karlsruhe 1968, Is.

17 Z. B. zeigt eine Uberprüfung des - wahllos herausgenommenen - BGHZ 40. Band, daß bei den ersten 107 Seiten - I7 Entscheidungen aus dem Jahre 1963 - etwa 8o Belege auf Autoren und nur 20 auf Gerichte verweisen - die Rechtsprechung des eigenen Gerichts und des Reichsgerichts ausgenommen. Ober den Einfluß der Lehre auf die Praxis gibe es auch bei KaupenRasehorn (N. Is) widntige Hinweise.

18 E. J. Cohn, Der obskure deutsche Professor, JZ 1967, 419.

$19 \mathrm{H}$. E. Henke, Wie tot ist die Begriffsjurisprudenz, Zeirschrift für Zivilprozeß 80 (L 967) S. I ff.

- So können dann derartige > Pannena passieren wie bei der Neufassung des $\$$ so Abs. = StGB im Gesetz über Ordnungswidrigkeiten (!), die zur Amnestie für NS-Mörder wird, vgl. Der Spiegel 22/1969, S. 33 .

-1 Vgl. auch Konrad Redeker, Bewältigung der Vergangenteit als Aufgabe der Justiz, NJW 1964, I097. 
Diskussionen und Polemiken, wie sie zur Zeit von Ernst Fuchs gang und gäbe waren und die gerade dessen Wirken, das Wirken der Freirechtsschule, ermöglichten”2. An diese heutige Juristenelite mag Dahrendorf denken, wenn er davon spricht, sie garantiere "die Verfassung des Rechts nur bedingt und die Verfassung der Freiheit gar nicht ${ }^{23}$. Gewiß, gegenüber demjenigen, der sich an den wissenschaftlichen Komment hält, erweist man noch Toleranz, repressive Toleranz; für den, der gerade hiergegen opponiert, fällt die Toleranz fort und es bleibt allein die Repression. Diese Opponenten werden nicht etwa als Irrlehrer bezeichnet, sie werden gar nicht ver-zeichnet, trotz aller Zitierwut unserer juristischen Creme nicht erwähnt und somit als Unpersonen im Rechtswesen behandelt ${ }^{21}$.

In dieser Zone des Schweigens liegt fast die gesamte Tätigkeit der Tatsachengerichte, insbesonderer der Amtsgerichte, obgleich diese $97 \%$ der Strafsachen und $87^{\%} \%$ der Zivilsachen erledigen ${ }^{24^{2}}$. Hierüber kann sich der Jurist im wesentlichen nur aus der Tagespresse, nicht aber aus der Rechtspresse informieren. Nicht einmal die Rechtssoziologen - Viehweg, Fechner, Hirsch, Rehbinder - interessieren sich für die Tärigkeit der richerlichen Praktiker, überlassen dieses Feld den reinen Soziologen"25. Nur die Ignoranz über die Arbeitsweise des Praktikers läßt den heftigen Methodenstreit über die Theorie von Viehweg, der Richter lasse sich mehr von Topoi als von dogmatischen Erkenntnissen leiten, verstehen ${ }^{26}$. Sollte sich hingegen ein Praktiker - was gewiß selten geschieht - in die fast jeden aktuellen Bezug ängstlich vermeidende Schrift Viehwegs - Topik und Jurisprudenz - vertiefen, so wird er, was er versteht, als Selbstverständlichkeiten, ja Platituden, bezeichnen und gerade deshalb, eingedenk der Kontroverse in der Rechtslehre, rätseln, wer hier der Scharlatan und wer der Dupierte ist. Ein Vergleich mit amerikanischen rechtssoziologischen Untersuchungen über die richterliche Tätigkeit zeigt, daß bei uns die Darstellung der Rechtspllege als solche - und nicht als angewandter Rechtstheorie - sich erst im vorwissenschaftlichen Stadium befinder ${ }^{27}$.

Die mangelnde Beachtung des eigenen Lagers für die Tätigkeit der überwiegenden Mehrheir der Richter ${ }^{28}$ - denen allerdings das Narcoticum der (natürlich nicht näher untersuchten) richterlichen Unabhängigkeit bereirwillig überlassen wird - muß bei dem Durchschnittsrichter einen Trend zur Unsicherheit und

: Vgl. Albert S. Foulkes, Ernst Fuchs und die Freirecheslehre, in Ernst Fuchs, Gerechrigkeitswissenschaft, Karisruhe 1965 Neuauflage S. 225.

23 R. Dahrendorf, Geselischaft und Demokratie in Deurschiand, 1965, S. 261.

94 Dies trifft selbst >rechte noch völlig juristisch gesonnene Prakriker wie Egon Schneider. Vgl. u. a. den Beitrag des Rechtsiehrers Zippelius zur Topik-Lehre. NJW 1967, 2229, wo der umfassende Beitrag von Schneider, Rechrspraxis und Rechrswissenschaft, MDR 1967,6, der dem Praktiker die Topik-Lehre begreiflich zu machen versucht, überhaupt nicht angeführt wird. Bei mir zeigt das Verschweigen schon groteske Züge: Der Band $\approx$ Im Namen des Volkes? ist in der DRiZ nicht einmal unter "Neue Bücher a aufgeführt, noch besprochen worden. Prof. Berges, Recht und Richter, Lehre und Praxis, DRiZ 1967, 290 setzt sid. fast ausschließlich mit mir in seinem langen Beitrag auseinander, ohne dies anzuzeigen. Der Bundesrichter Fledk weist in DRiZ ${ }_{1969,167}$ in einer kurzen Rezension auf die Neue Müller-Paperbadk-Reihe, Rechr-Justiz-Zeitgeschehen, hin, wobei ihm beim Aufführen der bislang erschienenen Autoren mein Name nicht in die Feder will, weshalb ich mich dann als einziger $* u$. a. a finden muß.

240 Zahlen bei Brune, DRiZ 1966, 370; Jahresbericht des Bundesjustizministeriums 1967, S. 13.

\$ Vgl. Kaupen a. 2. O. (N. 7) S. I 16 mit weiteren Belegen. Der unter N. 7 aufgeführte Sammelband enthält beinen Betrag eines deueschen Rechtssoziologen zur Rechrsprechung.

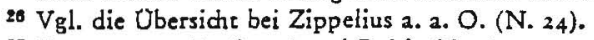

27 Kaupen a. a. O., S. 118 und Reich (N. 7 ).

$98 \mathrm{Vgl}$. im übrigen auch die ganz knappen Hinweise auf die Justiz bei dem fortschritrlichen Rechtsiehrer Rudolf Wiethölter, Recheswissenschaft, Fischer Bücherei, I968 S. 26-42 (An sich soilte der Titel ja $>$ Recht $\times$ lauten!) 

hervorrufen. So kommt es nicht zu einem echten Selbstverständnis, so kommt es nicht zu einer Kommunikation mit der Gesellschaft.

\section{Das Ende der Justiz?}

Und wie soll es weitergehen, weitergehen mir der Justiz und dem Richter? Gerade auf diese Frage hin habe ich den Beitrag von Rotrleuthner mit besonderer Spannung gelesen. Die von ihm eindringlich geschilderten Schwierigkeiten mit der heutigen Justiz waren im wesentlichen ja auch mir geläufig, ließen in mir das Bewußtsein einer Auswegslosigkeit entstehen, worüber ich mir durch Propagieren blasser Zukunftsziele hinwegzuhelfen suchte. Würde Rottleuthner von einer progressiv soziologischen Sicht her einen Ausweg aufzeigen? Hier sehe ich

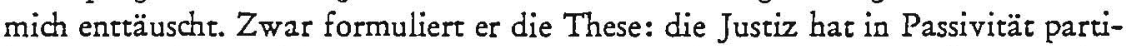
kulare soziale Konflikte zu lösen (S. I4); er spricht auch von einer kommunikativen, d. h. verbindlich argumentierenden Praxis (S. 26), was zweifellos Ansätze sind. Aber wie sind sie zu verwirklichen? Für diese sich aufdrängende Frage gelten seine beiden Schlußworte: er schweigt.

Aber auch dieses Schweigen ist eine Aussage. Es mag aus der von ihm geschilderten fehlenden Einbindung des Richters in die Gesellschaft sowie aus der von mir nochmals herausgestellten Randexistenz des Durchschnitrsrichters in seinem eigenen Bereich, im Rechrswesen, zu folgern sein, daß es überhaupt nicht mehr weitergeht, daß es also mit der Justiz zu Ende geht.

Dies ist meine These, nicht mehr unsere, also der Justizreformer insgesamt. Diese sind eigentlich optimistisch. Am optimistischen sind dabei - und dies überraschr natürlich nicht - die aus dem Wissenschaftsbereich kommenden: Gewiß - die gute alte Tante Justitia hat Fetr angesetzt, sie atmet auch etwas schwer; aber an sich ist sie kerngesund; mit einigen technischen Reformen ${ }^{29}$ und mit einem veränderten Selbstverständnis der Richter ${ }^{30}$ haben wir sie bald wieder in Schwung. Die Richterreformer sehen aufgrund ihrer leidgeprüften Erfahrung tiefer. Natürlich sind auch sie für technische Reformen - Beschleunigung der Prozeßverfahren, organisatorische Änderungen im Gerichtswesen. Vor allem aber wird nach mehr Demokratie gerufen - Richterwahl durch Parlamentarier, richterliche Selbstverwaltung, umlaufender Vorsitz im Kollegium, dissenting opinion ${ }^{31}$ - und auch ich habe einem großen Teil dieser Forderungen zugestimmt und werde es weiter tun - auch dort, wo es die zweitschlechtesten sind, denn sie können jedenfalls den derzeitigen Zustand verbessern. Aber letztlich kommt man um die Frage nicht herum: können mit derartig einfachen Mitteln die jedenfalls im Hinblidk auf das gesellschaftliche Leben im Jahre 2000 als völlig versteinerr zu bezeichnenden Strukturen der Justiz gelöst und geändert werden? Werden hier nicht lediglich die Wände eines Eimers geflickt, der schon längst keinen Boden mehr hat? Was den Reformern und Reformen fehlt, ist, die Justiz selbst in Frage zu stellen. Wer von der Exrigkeit einer Instirution ausgeht, wird auch immer zu dem Ergebnis kommen, daß sie ewig sei. Dabei ist unsere Justiz nicht wie die katholische Kirche 2000 Jahre, sondern nur ein kurzes Jahrhundert alt. So wie ihre Strukturen

29 H. P. Bull, Der Richeer - sköniglich «, demokratische oder was sonst?, ZRP 1968, 69.

so Raiser, a. a. O., (N. 5) S. 400 . Vgl. insbesondere den völlig auf der Linie einer Obrigkeitsjustiz liegenden Vortrag von Prof. Friesenhahn auf dem III. Internationalen Richterkongreß, Der Richter in unserer Zeit, DRiZ $1969,169$.

31 Rasehorn in *Im Namen des Volkes? \&. 185. 
heute sind, hat sie sich der Obrigkeitsstaat geschaffen. Durch richterliche Beamte übte er in einem formal-legalen oder auch primitiv-moralischen Rahmen Kontrolle über den Bürger aus, wobei durchaus ein Bedürfnis nach rechtlicher und selbständiger Gestaltung der Beziehungen der Staatsbürger untereinander - und in gewisser Hinsicht auch gegenüber der Staatsgewalt selbst - in einer für die Obrigkeit unschädlichen Weise befriedigt werden konnte. Mit dem Untergang des Obrigkeitsstaates ist auch für dessen Justiz kein Raum mehr, wobei es bizarr - aber zugleich symptomatisch - ist, daß dieser Untergang mit Demonstrationsprozessen eingeläutet wird, also Verfahren aufgrund jener Strafnormen, mit denen der Obrigkeitsstaat und seine Justiz am stärksten zusammengekettet sind.

An dieser Stelle ruft mich indes Lenk zur Ordnung, da ihm ähnliche, in unserem Band allerdings nur vage ausgeführte Gedanken als irreal erscheinen. Richtig ist, $\mathrm{da} ß$ unsere auf einen Obrigkeitsstaat ausgerichtete Führung mit Exekutive und Justiz noch viel Unheil anrichten können und daß es Tendenzen gibt - angezeigt in der Notstandsgesetzgebung oder in der Justiz durch die fortschreitende Bürokratisierung -, die einem Absterben von Obrigkeitsstaat und -justiz widersprechen. Gleichwohl läßt sich zeigen, daß der Staat als Eigenwesen - als Nation oder als Volk (Hitler: „Du bist nichts, Dein Volk ist alles.«) - im gesellschaftlichen Bewußtsein immer stärker verblaßt und daß im Rechtsbereich nicht mebr abstrakte Verbote und formale Kriterien, wie noch eine Generation zuvor, selbstverständlich befolgt werden. Hiernach ließe sich in verfolg der Systemanalyse von Talcotr Parsons davon ausgehen, daß das bisherige integrative System durch ein »offenes« abgelöst wird, als universalistischer Markt, mit flexibler Anpassung, eine Konstellation mit dem Instrument der Kontrolle durch Massenkommunikationsmittel, die im engen Kontakt zu den Prozessen des täglichen Lebens stehen und bereit sind, jedes neue Stimulans, jede neue Anregung an das Publikum weiterzuleiten. In einer derartigen Gesellschaft erweist sich die Justiz - auch eine Justiz nach den Ideen der Reformer - als zu starr und zu wenig effektiv.

Die moderne, von der Technik her organisierte, im übrigen aber im Sinne einer Herrschaftsverwaltung eher anarchische Gesellschaft wird in erheblichem Umfang mehr Betreuungspersonal - mehr Psychotherapeuthen, mehr Sozialhelfer gebrauchen. Aber es wird auch Konflikte geben, die weder durch psychische, noch durch organisatorische Einwirkung zu lösen sind. Hier ist Raum für den Richter oder für den Nachfolger des Richters. Er muß also von der bisherigen Stabsfunktion zur Betreuungsfunktion hinüberwechseln, wobei auf das eingangs Gesagte zu verweisen ist, darauf, wie sehr schon dem heutigen Richter Betreuungsfunktion zufallen, d.h. echte Verantwortung für den Rechtsuchenden als Mitmenschen.

Dies bedeutet weiter eine Ausrichtung des Richters nach dem Rechtsanwalt - der in informeller Weise zu versuchen hat, Streit zu schlichten und soziale Konflikte zu beseitigen -, während heure der Rechrsanwalt umgekehrt als "Organ der Rechtspflege« sich der Justiz anhängt. Selbstverständlich, daß der Richter entbürokratisiert werden muß, womit er sich letztlich anderen Rechtspflegeberufen - Rechtsanwalt, Notar, Gerichtsvollzieher, Bewährungshelfer - nur angleidht. Er wird ähnlich wie der Schiedsmann heute in den Großstädten eine Stadrvierrelzuständigkeit haben und in überschaubaren, privaten Gebäulichkeiten mit wenigen Hilfspersonen am $»$ Runden Tisch « das Gespräch mit Rechrssuchenden führen $^{32}$. Es ist weiter eine Selbstverständlichkeit, daß die Ausbildung eines derar-

32 v. Münchhausen a. a. O. (N. 13) hält eine solche Lösung für verfassungsrechtlich unzulässig; es kann aber letztlich nur der hier einen Verstoß gegen Art. 92 GG erblióren, der die Justizbürokratie unter Verfassungsschurz stellt. 
tigen Richters völlig von der heutigen Juristenausbildung abweichen, nur zu $10 \%$ eine juristische und im übrigen eine sozialpsychologische sein wird.

Schauen wir uns die derzeitigen Gerichtsinstitutuionen nach der Ausrichtung auf dieses Modell hin an, so werden wir in der Person und in der Tätigkeit des Richters an Ein-Mann-Gerichten eine gewisse Praedestinierung erkennen ${ }^{33}$. Man kann durchaus noch weitergehen: eine Beseitigung der - vom Justizapparat und der Rechtsdogmatik gebildeten - Machtstrukturen im Rechtswesen läßr Formen zurüds, die zu einer Gesellschaftsrelevanz ausgebaut werden können. Dies gilt auch für die Bundesgerichte als Gremien, die im formalrechtlichen Bereich in Zusammenarbeit und Ergänzung zu den Gesetzgebungsteams Lösungen für Sozialund Wirtschaftsfragen entwickeln werden. Erst recht gilt dies für das Bundesverfassungsgericht als ein - von direkten Einflüssen - freies Kontrollorgan zu Uberprüfung von Akten der Administration auf ihre Demokratiegerechrigkeit, was insbesondere Minderheitenschutz bedeutet ${ }^{34}$.

Die verschiedenen Gerichte werden indes kaum noch etwas gemeinsam haben - es sei denn, daß ihre Entscheidungen Beschlüsse oder Urteile heißen - wie sie heute auch wenig gemeinsam haben, wobei allerdings ein bürokratischer Rechtspflegeapparat - zur hierarchischen Machtausübung von oben nach unten - die Verbindung herstellt. Gewisse Rudimente der Justiz mögen sich noch in einer Mittelstufe halten, die also Entscheidungen der ersten Instanz - eng! - auf einen Rechtsirrtum überprüft; eine solche Basis gibt indes einer Obrigkeitsjustiz keinen Soielraum zur Machtentfaltung mehr.

Obgleich die hier gezeigten Gerichtsformen von den vorhandenen niche sehr abweichen, wäre der optimistische Schluß verfehlt, wir befänden uns auf dem besten Wege. Das Gegenteil ist richtig. Tatsächlich zeigt sich bei der Justiz ein progressiv verlaufender Prozeß der Erstarrung in eine von den Maximen Perfektion, Zentralisierung und Spezialisierung profitierende Bürokratie. Symptomatisch sind die Maßnahmen zur Auflösung der - hier gerade als Keim eines künftigen Richtertyps gesehenen - kleinen Amtsgerichte, wobei sich aus den Begründungen unschwer das Ziel einer Verfestigung der derzeitigen Obrigkeitsjustiz entnehmen läßt ${ }^{35}$ : "Eine omnipotente Verwaltung ist emsig dabei, das Kanalbett zu zementieren ${ }^{36}$. Nach dem im politischen Leben längst als untauglich erkannten Subsidiaritätsgrundsatz des konservativen Publizisten Konstantin Frantz werden dem Richter Aufgaben genommen, die angeblich zu schematisch seien, und Rechtspflegern und Verwaltungsbeamten zugeteilt. Das kommt bei Verkehrswidrigkeiten so weit, daß die Verwaltung darüber befindet, was Ordnungsunrecht und was kriminelles Unrecht ist ${ }^{37}$. So wird gerade auf dem gesellschaftlich wichtigen Verkehrsrechtssektor der Richter in das zweite Glied gedrängt, vom Alltag der Gesellschaft entfernt. Auf diesem Sektor gibt es auch keine Opposition von, teilweise fortschrittlichen, Richterverbänden. Den Berufsverbänden - Gewerkschaften - ist die Zentralisierung und Bürokratisierung des Gegenspielers durchaus recint, wird hierdurch doch ihr Einfluß auf die eigenen Mitglieder und deren Zusammenhalt gestärkt. Hier geht es ihnen nur noch darum, im Bereiche

93 Vgl. hierzu auch Rasehorn, Ein Plädoyer für das kleine Amtsgeriche, erscheint demnäcist in $\operatorname{der} \mathrm{JZ}$.

34 Ablehnend neuerdings, wenn zuch ohne neue Gedanken, Wolfgang Birke, Richterliche Rechtsanwendung und geselischatliche Auffassungen, Köln I968, S. 22 ff.

35 H. H. Weyel, Aufhebung yon Kleingerichten im Bereiche der ordentlichen Gerichtsbarkeit, JVBl. 1967, 25; Auszug aus der Amtlichen Begründung für Hessen, DRiZ 1969, 24. Hierzu krivisch Kaupen-Rasehorn a. a. O. (N. Is).

36 Joachim, 3. 3. O. (N. S) S. 22.

37 W. Knode, Ein Exekutivstaac breitet sich aus, Vorgänge I/69 S. Ig. 
der Bürokratie mitzubestimmen, was hinter manchen Demokratisierungsbestrebungen der Richterverbände steht ${ }^{38}$. So wird nicht selten im Namen der Demokratie die Obrigkeitsjustiz auch von progressiven Richtern am Leben erhalten und damit der Weg für die Zukunft des Richters versperrt.

So mag der hier aufgezeigre künftige Richter ganz andere Wurzeln haben, sich vielleicht aus Schiedsmann oder Bewährungshelfer, vielleicht aber auch aus dem Rechtspfleger oder aus dem Beamten, der heute die Bußgeldbescheide erläßt, entwidkein. Dafür gibt es gerade in Deutschland ein Präzidenz: im Entstehen der Obrigkeitsjustiz selbst. Sie ist ja nicht aus den mitrelalterlichen Schöffengerichten erwachsen, sondern aus den nichtrichterlichen "gemieteten doctores" des römischen Rechts als Höflinge der Fürsten, die erst neben und dann anstelle der Schöffenrichter amrierten.

Aber müssen wir noch heute derartige zeitraubende Umwege gehen? 